\title{
The rise and spread of a new pathogen: Seroresistant Moraxella catarrhalis
}

\author{
Thierry Wirth, ${ }^{1,2,6,7}$ Giovanna Morelli, ${ }^{3,6}$ Barica Kusecek, ${ }^{3}$ Alex van Belkum, ${ }^{4}$ \\ Cindy van der Schee, ${ }^{4}$ Axel Meyer, ${ }^{1}$ and Mark Achtman ${ }^{3,5,7}$ \\ ${ }^{1}$ Department of Biology, University Konstanz, D-78457 Konstanz, Germany; ${ }^{2}$ Department of Systematics and Evolution, Muséum \\ National d'Histoire Naturelle, Ecole Pratique des Hautes Etudes, F-75231 Paris cedex 05, France; ${ }^{3}$ Department of Molecular \\ Biology, Max-Planck Institut für Infektionsbiologie, D-10117 Berlin, Germany; ${ }^{4}$ Department of Medical Microbiology and \\ Infectious Diseases, Erasmus MC University Medical Centre Rotterdam, 3015 GD Rotterdam, The Netherlands; ${ }^{5}$ Department of \\ Microbiology, University College Cork, Cork, Ireland
}

\begin{abstract}
The nosocomial human pathogen Moraxella catarrhalis is one the most important agents of human respiratory tract infections. This species is composed of two distinct lineages, one of only moderate virulence, the so-called serosensitive subpopulation, and a second, the seroresistant one, which is enriched among strains that harbor two major virulence traits: complement resistance and adherence to epithelial cells. Using a suite of population genetics tools, we show that the seroresistant lineage is also characterized by higher homologous recombination and mutation rates at housekeeping genes relative to its less pathogenic counterpart. Thus, sex and virulence have evolved in tandem in M. catarrhalis. Moreover, phylogenetic and Bayesian analyses that take into account recombination between the two clades show that the ancestral group was avirulent, is possibly 70 million years old, and must have infected mammals prior to the evolution of humans, which occurred later. The younger seroresistant isolates went through an important population expansion some 5 million years ago, coincident with the hominid expansion. This rise and spread was possibly coupled with a host shift and the acquisition of virulence genes.
\end{abstract}

[Supplemental material is available online at www.genome.org. The multilocus haplotypes are publicly available at http://web.mpiib-berlin.mpg.de/mlst.]

Human pathogens present ongoing challenges to public health. Recently, formerly commensal species have raised serious health concerns because of dramatic changes in their genetic makeup. This is the case for Moraxella catarrhalis, which has recently been reclassified from representing an emerging pathogen to an established nosocomial pathogen (Verduin et al. 2002). M. catarrhalis is a Gram-negative, aerobic diplococcus frequently found as a commensal of the upper respiratory tract (Johnson et al. 1981), but it is increasingly associated with acute otitis media and is associated with infectious exacerbations of chronic obstructive pulmonary disease (Hager et al. 1987; Murphy 1998). It is also the third most commonly isolated pathogen from the lower respiratory tract, after Streptococcus pneumoniae and Haemophilus influenzae. In immunocompromised hosts, M. catarrhalis can also cause pneumonia, endocarditis, septicemia, and meningitis (Catlin 1990; Daoud et al. 1996). Moreover, recent hospital outbreaks of respiratory disease due to M. catarrhalis (Patterson et al. 1988; Richards et al. 1993) have resulted in an expanding literature describing the agents and mechanisms of $M$. catarrhalis virulence (Helminen et al. 1993; Aebi et al. 1998; Boel et al. 1998).

Sequences of $16 \mathrm{~S}$ and $23 \mathrm{~S}$ rRNA, plus fingerprinting of outer membrane protein sequences, revealed that M. catarrhalis is composed of two distinct lineages (Bootsma et al. 2000; Stutzmann Meier et al. 2005) that differ in their potential for virulence; we

\footnotetext{
${ }^{6}$ These authors contributed equally to this work.

${ }^{7}$ Corresponding authors.

E-mail m.achtman@ucc.ie; fax 49-30-28460750.

E-mail wirth@mnhn.fr; fax 33-1-40-79-33-37.

Article published online before print. Article and publication date are at http:// www.genome.org/cgi/doi/10.1101/gr.6122607.
}

refer to them here as the seroresistant and serosensitive populations. The seroresistant population is strongly associated with two virulence traits: complement resistance and the ability to adhere to epithelial cells. Adherence is found in $92 \%$ of seroresistant isolates and is virtually absent in the serosensitive isolates (Bootsma et al. 2000).

Public health concerns create an urgent need to understand the effects of virulence factors, pathogenicity, and ecological niches on bacterial population structure and demography. Reconstructing the evolution of virulence within a bacterial species requires a global understanding of how that species has evolved. $M$. catarrhalis provides a tractable system for understanding the forces and processes that have shaped the evolution and origin of increased virulence because it displays a dichotomous pathogenic pattern as well as moderate epidemiological complexity and genetic diversity. However, the population genetic structure and ongoing gene flow between lineages have not been elucidated in $M$. catarrhalis (and many other bacterial species) until now, partly because phylogenetic tools that are used to analyze the evolution of sequence diversity are not designed to cope with the extensive genomic re-shuffling caused by homologous recombination (Feil et al. 2001).

Multilocus sequence typing (MLST) provides a uniform, expandable typing method that can be used for long-term epidemiology (Maiden 2006). We have developed such an MLST scheme for $M$. catarrhalis that is based on the sequences of eight housekeeping gene fragments. This scheme is freely available for interrogation and data submission via a public Web site (http:// web.mpiib-berlin.mpg.de/mlst). In this study, we analyzed their ancestral relationships and the evolution of virulence by using a 
suite of population genetic tools on multilocus sequence data from a global sample of strains from symptomatic and asymptomatic infections. The analytical approaches included reconstructing the diversity of ancestral sequences prior to recombination (STRUCTURE) (Falush et al. 2003a), reconstructing tree topologies after removing recombinant sequences ("tree purging") (Wirth et al. 2006), and unraveling distinct demographic histories in the two main populations with the help of Bayesian coalescence approaches (Pybus and Rambaut 2002). Our results demonstrate that the seroresistant population underwent a rapid population expansion during the last 5 million years (Myr), approximately the same time at which it acquired virulence traits; this population has also undergone extensive recombination. In contrast, the serosensitive population is much older, has undergone less dramatic demographic changes, and possesses a population structure that is more clonal. Thus, the most admixed population is also the most virulent, whereas genetic admixture is rare among strains that lack complement resistance and adherence to epithelial cells, suggesting that sex and virulence are causally related.

\section{Results}

\section{Sequence analyses}

We assembled a collection of $268 \mathrm{M}$. catarrhalis of diverse geographic origins, half of which had been isolated from symptomatic patients and the other half from asymptomatic people (Supplemental Table S1). We designed primers to amplify and sequence fragments of eight housekeeping genes that are distributed around the M. catarrhalis genome (Supplemental Table S2) and sequenced these fragments from all strains. Each unique sequence was assigned a distinctive allele number, and each unique combination of alleles was assigned a distinctive sequence type (ST) number. A total of 173 STs were found among the 268 strains (Supplemental Table S1). We did not detect any obvious geographical clustering of individual strains within STs that con- tained more than one isolate, and we could not identify any other evidence for biogeographic specificity.

The phylogenetic analysis described below assigned 42 strains to a "serosensitive" population and the remaining 222 to a "seroresistant" population. However, four strains differed markedly from the others and may belong to a separate species or subspecies. Three of these strains (all in ST116) were isolated in Ethiopia and the fourth (ST127) in the United States. ST116 and ST127 differ in the alleles at all eight genes except for $a d k$, but sequence alignments of the concatenated sequences showed that they have a common ancestry and are only distantly related to $M$. catarrhalis (Supplemental Fig. S1). Of the eight gene fragments, only ppa clusters among sequences from $M$. catarrhalis, intermingled with sequences from serosensitive strains, suggesting at least one historical interspecies homologous recombination. The mean genetic distance (Tamura-Nei) between sequences from this species/subspecies and the $M$. catarrhalis cluster is 0.140 ( \pm 0.009$)$, whereas the genetic distance between the two clades within $M$. catarrhalis is only $0.044( \pm 0.003)$. These data suggest that these four strains are related to $M$. catarrhalis but sufficiently distinct that they can be used as outgroups (Supplemental Fig. S2) but should not otherwise be included in phylogenetic analyses of diversity within $M$. catarrhalis. We ignore them in the following descriptions.

\section{Polymorphism and selection}

The mean pairwise nucleotide diversity $(\pi)$ was higher within the serosensitive population than within the seroresistant population $(0.025$ vs. $0.01 ; P<0.01)$, suggesting that the serosensitive gene pool is older than its seroresistant counterpart (Table 1). Both populations possess a similar number of alleles, although the seroresistant population includes five times as many strains as does the serosensitive population, supporting this inference. The number of alleles found in the seroresistant population varies with the locus (range: 3-38), perhaps reflecting different levels of purifying selection. We investigated this possibility by a slid-

Table 1. Population genetic analyses of the serosensitive $(n=42)$ and seroresistant $(n=222)$ populations

\begin{tabular}{|c|c|c|c|c|c|c|c|c|c|}
\hline \multirow[b]{2}{*}{ Gene } & \multirow{2}{*}{$\begin{array}{l}\text { Length } \\
\text { (bp) }\end{array}$} & \multirow{2}{*}{$\begin{array}{c}\text { No. of } \\
\text { haplotypes }\end{array}$} & \multirow{2}{*}{$\begin{array}{c}\theta \\
\text { (total) }\end{array}$} & \multicolumn{3}{|c|}{$\pi$} & \multirow[b]{2}{*}{ Tajima's D } & \multirow[b]{2}{*}{$\mathrm{Fu}^{\prime} s F_{\mathrm{s}}$} & \multirow[b]{2}{*}{$K_{\mathrm{a}} / K_{\mathrm{s}}$} \\
\hline & & & & Total & Synonymous & Nonsynonymous & & & \\
\hline \multicolumn{10}{|c|}{ Serosensitive populations } \\
\hline glybeta & 537 & 24 & 0.0428 & 0.0412 & 0.1965 & 0.0046 & -0.1421 & 0.201 & 0.0218 \\
\hline ppa & 393 & 22 & 0.0509 & 0.0553 & 0.2661 & 0.0063 & -0.0256 & 0.926 & 0.0205 \\
\hline efp & 414 & 15 & 0.0140 & 0.0119 & 0.0510 & 0.0000 & -0.5530 & -1.992 & 0.0000 \\
\hline $\operatorname{trp} E$ & 372 & 11 & 0.0112 & 0.0081 & 0.0313 & 0.0012 & -0.9259 & -1.393 & 0.0388 \\
\hline fumC & 465 & 15 & 0.0230 & 0.0259 & 0.1063 & 0.0017 & 0.1204 & 2.234 & 0.0153 \\
\hline mutY & 426 & 21 & 0.0262 & 0.0260 & 0.0711 & 0.0121 & -0.1646 & -1.672 & 0.1684 \\
\hline$a b c Z$ & 429 & 22 & 0.0227 & 0.0143 & 0.0388 & 0.0072 & -1.3314 & $-6.738^{*}$ & 0.1792 \\
\hline$a d k$ & 471 & 16 & 0.0222 & 0.0153 & 0.0475 & 0.0054 & -1.1488 & -0.844 & 0.1106 \\
\hline Mean & 438.4 & 18.2 & 0.0266 & 0.0247 & 0.1011 & 0.0048 & -0.5214 & -1.1597 & 0.0693 \\
\hline \multicolumn{10}{|c|}{ Seroresistant populations } \\
\hline glybeta & 537 & 38 & 0.0134 & 0.0171 & 0.0588 & 0.0052 & 0.7384 & $-3.026^{*}$ & 0.0873 \\
\hline ppa & 393 & 17 & 0.0047 & 0.0075 & 0.0356 & 0.0000 & 1.3589 & -1.805 & 0.0000 \\
\hline efp & 414 & 11 & 0.0105 & 0.0029 & 0.0116 & 0.0002 & $-1.9952^{*}$ & $-2.795^{\star}$ & 0.0171 \\
\hline $\operatorname{trp} E$ & 372 & 3 & 0.0009 & 0.0001 & 0.0001 & 0.0000 & -1.2715 & $-5.266^{*}$ & 0.3019 \\
\hline fumc & 465 & 13 & 0.0076 & 0.0068 & 0.0231 & 0.0016 & -0.2996 & 0.637 & 0.0679 \\
\hline mutY & 426 & 27 & 0.0122 & 0.0206 & 0.0695 & 0.0055 & 1.7120 & 0.794 & 0.0776 \\
\hline$a b c Z$ & 429 & 25 & 0.0129 & 0.0171 & 0.0519 & 0.0072 & 0.7454 & 0.287 & 0.1313 \\
\hline$a d k$ & 471 & 22 & 0.0089 & 0.0069 & 0.0135 & 0.0049 & -0.6167 & $-4.053^{*}$ & 0.3600 \\
\hline Mean & 438.4 & 19.5 & 0.0089 & 0.0099 & 0.0330 & 0.0031 & 0.0465 & -1.903 & 0.1304 \\
\hline
\end{tabular}

${ }^{*} P \leq 0.05$. Corrections for multiple tests were not applied.

\section{Genome Research}

www.genome.org 


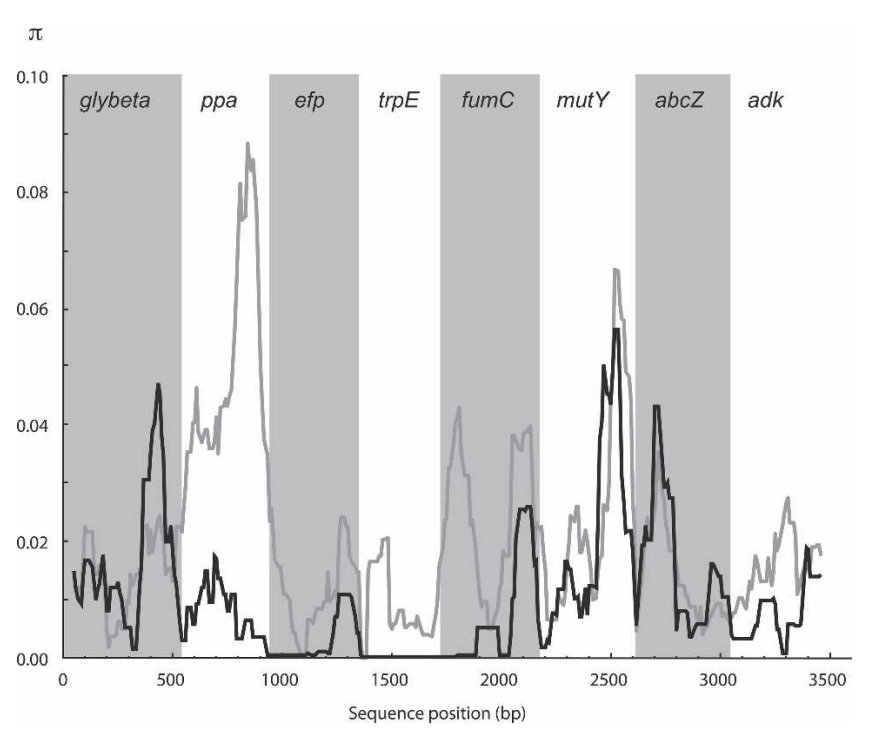

Figure 1. Sliding-window analysis of polymorphism for the eight concatenated housekeeping genes. (Light gray) Serosensitive; (black) seroresistant.

ing-window analysis of the total pairwise nucleotide diversity (Fig. 1), which showed substantial local variation in the degree of divergence in both serosensitive and seroresistant populations, some of which were congruent between both populations.

MLST relies on the analysis of core genome housekeeping genes under the assumption that they are under purifying selection. The observed peaks in diversity at certain positions in Figure 1 might reflect a relaxation of selective constraints at those positions or might reflect positive selection. Positive selection might significantly affect data interpretation and lead to unreliable phylogenetic reconstructions, but is thought to be rare (Zhang and Li 2005). We therefore tested this possibility with three population genetic tests of selection, Tajima's $D$ statistic (Tajima 1989), Fu's $F_{\mathrm{s}}$ statistic (Fu 1997), and the $K_{\mathrm{a}} / K_{\mathrm{s}}$ test. None of the tests produced significant evidence of selection within the serosensitive population, except for a significant Fu test for the $a b c Z$ gene $(P<0.05)$ (Table 1$)$. In contrast, in the seroresistant population, four out of the eight genes displayed significantly negative values of Fu's $F_{\mathrm{s}}$. Fu's $F_{\mathrm{s}}$ statistic is a one-sided test for an excess of rare alleles, a typical signature for population expansion as well as for selection. If the negative values of Fu's $F_{\mathrm{s}}$ reflected positive selection (or genetic hitchhiking), they should be accompanied by signals in the $K_{\mathrm{a}} / K_{\mathrm{s}}$ ratio and Tajima's $D$. However, the $K_{\mathrm{a}} / K_{\mathrm{s}}$ ratio, which measures the ratio of nonsynonymous to synonymous substitution rates, was $\ll 1.0$ for both $M$. catarrhalis lineages (Table 1), a value that is characteristic of purifying selection. And there was no support for positive selection in Tajima's $D$. Thus, we infer that the negative values of Fu's $F_{\mathrm{s}}$ in the seroresistant population may reflect population expansion.

\section{Phylogenetic analyses}

Prior to drawing any phylogenetic inferences, we first inferred the quality of the phylogenetic information contained in the data by plotting transition and tranversion rates as a function of genetic distances (Fig. 2). This graph shows that neither transversions nor transitions are saturated but, rather, both increase linearly with increasing genetic distance. We then measured substitution saturation using the Xia index (Xia et al. 2003) for all three codon positions. The observed $I_{\text {ss.c }}$ value of 0.848 was significantly larger than the $I_{\mathrm{ss}}$ value of 0.836 , thus confirming that there is little saturation at these sites.

Neighbor-joining, maximum-likelihood, and Bayesian trees were then constructed independently for the concatenated sequences of all eight gene fragments. All three phylogenetic methods produced very similar trees containing two major monophyletic clades (Fig. 3A), which we refer to as populations. The statistical support for these groups is very high, with $100 \%$ bootstrap values. The same groupings were obtained when we built phylogenetic trees including the four outlier strains (Supplemental Fig. S2). Rooting the tree with those outliers as an outgroup confirmed that the two main clades are monophyletic and that neither is an immediate ancestor of the other (Supplemental Fig. S3).

Figure 3A includes a couple of strains with long branches in the serosensitive grouping. These strains were not significantly different from others in that grouping based on the STRUCTURE analyses and were therefore included in our demogenetic analyses. An alternative possibility that we have not definitively excluded is that these strains represent highly diverse bacteria from rare or undersampled populations such as described for Escherichia coli (Wirth et al. 2006), which would reduce our estimates of age and have an impact on the demographic parameter estimates.

The dichotomy into two clades also corresponded well, albeit not perfectly, with bacterial sensitivity to serum complement-mediated lysis. Twenty-five of 29 (86\%) serosensitive strains were assigned to the serosensitive population, whereas $53 / 54(98 \%)$ seroresistant strains were in the seroresistant population (Supplemental Table S1). Similar patterns were also observed for the three $16 \mathrm{~S}$ rRNA types: three of four type 2 strains and all three type 3 strains were assigned to the serosensitive population, whereas all seven type 1 strains were in the seroresistant population $\left(\chi^{2}=10.94, \mathrm{df}=2, P \leq 0.01\right)$.

An occasional lack of correspondence between serum resistance or rRNA type with population might reflect recombination. We first tested whether recombination had been frequent by split decomposition. Split decomposition calculates networks of multiple alternative pathways between taxa whenever homoplasy or recombination results in phylogenetic inconsistency. The analysis of the concatenated genes recovered the same phylogenetic

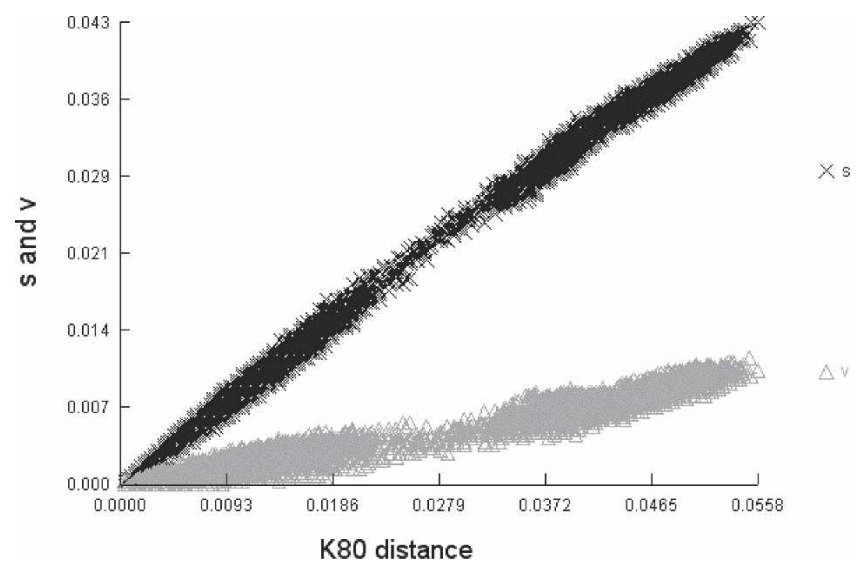

Figure 2. Plots of transitions (black crosses) and transversions (gray triangles) versus genetic distance (Kimura two-parameter model; K80) for eight concatenated sequences. There is no apparent saturation. 


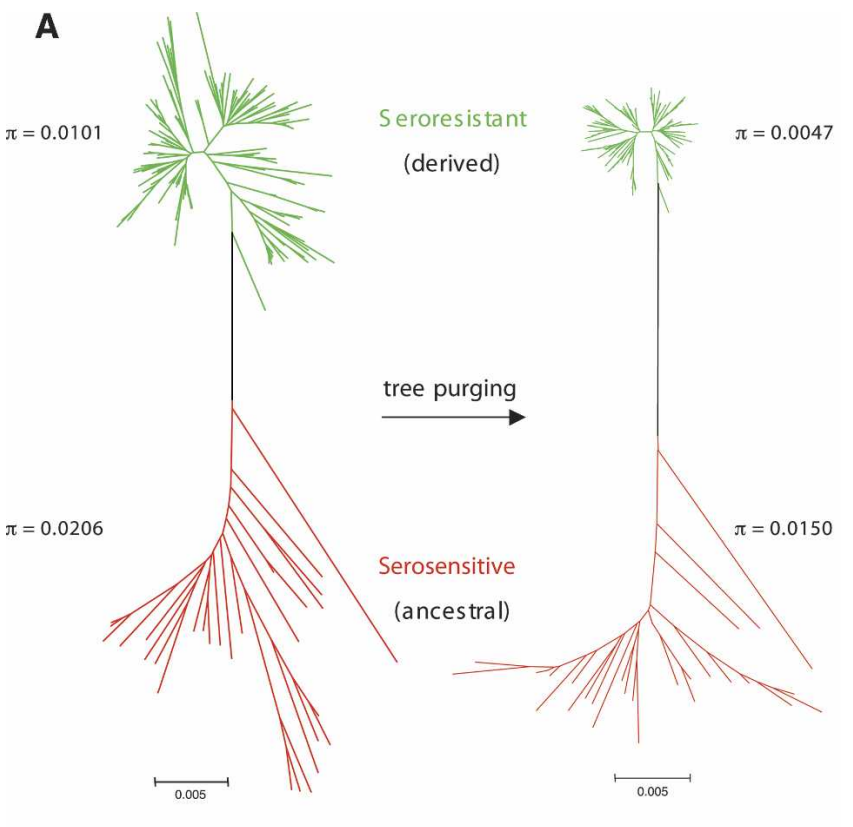

B

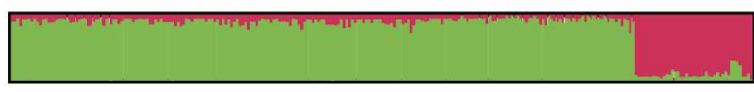

C

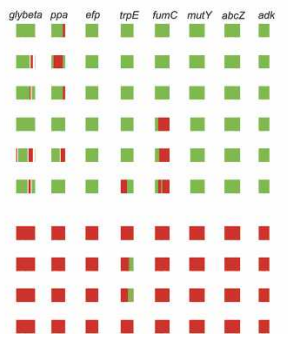

Figure 3. Phylogeny and ancestry of $264 \mathrm{M}$. catarrhalis isolates. (A) Unpurged (left) and purged (right) neighbor-joining trees of concatenated sequences using Tamura-Nei $+\mathrm{G}+$ I models. The bootstrap support for the two colored groups is $100 \%$. Unpurged tree model: $G=0.412, I=0.828$; purged tree model: $G=0.353, I=0.839$. (B) Proportions of ancestry from two ancestral populations as inferred by the linkage model of STRUCTURE. This plot shows one vertical line for each isolate in which the proportions of ancestry from the two sources are color-coded. Only recombinations between the two populations can be detected. (C) Examples of recombination within 10 random strains belonging to the two main populations. Boxes colored as in $A$ indicate sources of ancestry of stretches of nucleotides within each of the eight loci, shown in the order glybeta, ppa, efp, trpE, fumC, mutY, abcZ, and adk. Note that the serosensitive strains (bottom) are genetically rather homogeneous, whereas the seroresistant strains (top) are more admixed.

clusters as the traditional phylogenetic approaches (Fig. 4). Reticulations are common in the seroresistant population but rarer in the serosensitive population, suggesting that recombination has been more frequent in the former. Interclade recombination may have also occurred because many reticulations were detected between the two populations and near the base of the seroresistant clade. Import of sequences from the serosensitive clade into the seroresistant clade is also supported by the sequence alignment (Supplemental Fig. S1) wherein multiple short stretches of DNA in the seroresistant clade have a serosensitive origin. These short stretches are particularly obvious for the glybeta gene fragments.

\section{Population genetics and recombination}

Split decomposition is useful for visualizing alternative possible evolutionary relationships between sequences but does not provide details of individual recombination events, nor does it assess their statistical support. We therefore analyzed polymorphisms within the eight gene fragments with STRUCTURE (Pritchard et al. 2000; Falush et al. 2003a), a Bayesian method to identify the ancestral sources of nucleotides within $K$ groups of recombining organisms. The ancestry of each strain can be estimated as the summed probabilities of derivation from each group over all polymorphic nucleotides. STRUCTURE recognized two groups of strains within M. catarrhalis that were largely homogeneous in terms of their ancestral sources of polymorphism. The groups are completely congruent with those from the phylogenetic approaches (Fig. 3B). However, a closer look revealed that numerous strains, particularly those within the seroresistant population, are of mixed ancestry, whereas the serosensitive strains are far more homogeneous (Fig. 3C). Virtually all M. catarrhalis strains possess some transferred nucleotides, but it appears that the seroresistant mosaicism is largely due to homologous recombination with the other group, therefore confirming the observed pattern in the split decomposition analysis.

The STRUCTURE approach also does not provide quantitative estimates of the relative frequency of homologous recombination within and between groups. We therefore calculated the composite likelihood of $r / \mu$ (McVean et al. 2002), the relative probability that any single nucleotide will be switched because of mutation versus recombination (Table 2). This test confirmed our previous conclusions; the estimates of $r / \mu$ are much lower in the serosensitive population (0.625) than in the seroresistant one (3.833). A significant signature for recombination was found in only four out of eight housekeeping genes in the serosensitive population, whereas all eight gene fragments displayed signatures of recombination events in the seroresistant population. Furthermore, the mean $r / \mu$ ratios were approximately six times higher in the seroresistant population, showing that mutation is the main driving evolutionary force in the serosensitive population, whereas recombination is more prevalent in the other population (Table 2).

Considering these results, we attempted to deduce the true branching order and the true branch lengths by purging recombinant sites. After purging, the two populations remained distinct, but the topology of the neighbor-joining tree changed noticeably (Fig. 3A). The genetic diversity within the serosensitive population remained largely unaffected, whereas the branch lengths shortened dramatically and the estimates of $\pi$ dropped twofold in the seroresistant population (Fig. 3A). This indicates that the seroresistant population is much younger than the serosensitive population, which would not have been as clear had we not corrected for recombination.

\section{Demographic history}

We analyzed the mismatch distributions (frequencies of pairwise differences between haplotypes) in order to evaluate whether recent population growth has occurred in the two populations (Fig. 5). The serosensitive population has an extremely ragged distribution, which is multimodal and does not fit a sudden expansion model according to the Harpending raggedness index

\section{Genome Research}

www.genome.org 


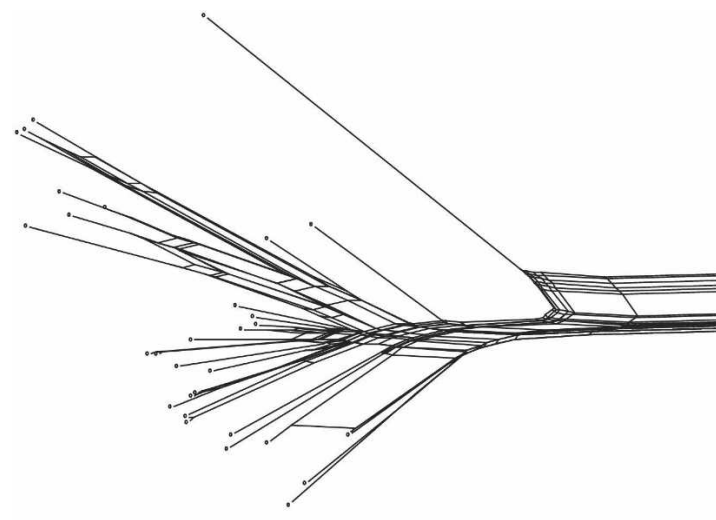

Serosensitive $M$. catarrhalis

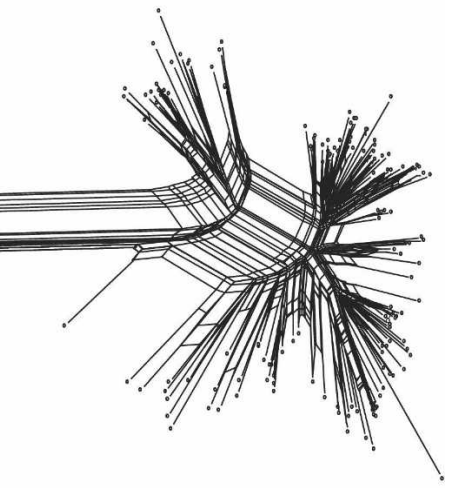

Seroresistant $M$. catarrhalis

Figure 4. Split decomposition analysis of eight concatenated housekeeping genes.

the age of the MRCA of the seroresistant population, which dates back to $6 \mathrm{Myr}$ (purged)-17 Myr (raw). The importance and the impact of the purging process are clearly illustrated by the generalized skyline approach. Without correction, the age of the seroresistant population would have been overestimated by almost threefold, and the strength of a demographic expansion that began some 4 Mya would have been strongly underestimated. Without correction, the seroresistant population size increased by a factor of 60 (from $\sim 10^{7}$ to $\left.6 \times 10^{8}\right)$, whereas after purging, the population expanded $>4000$-fold from $\sim 10^{6}$ to $4 \times 10^{9}$. The situation is exactly the opposite for the serosensitive strains, where the time of the MRCA is slightly underestimated and the growth rate is overestimated

$(P=0.001)$. In contrast, the mismatch distribution in the seroresistant population resembles the bell curves that are characteristic of a recent demographic expansion (Harpending et al. 1998), and the hypothesis of expansion could not be rejected $(P=0.567)$. Based on a stepwise expansion model, the effective population size of the seroresistant population increased by a factor of $13\left(\theta_{0}=12.799\right.$ and $\left.\theta_{1}=168.438\right)$ with a $\tau$-value of 28.633 (95\% interval confidence 20.047-53.254). Assuming the same mutation rate $(\mu)$ as for $E$. coli $\left(7.6 \times 10^{-10}\right.$ per nucleotide/ yr) (Wirth et al. 2006), the expansion event took place $\sim 5.3$ million years ago (Mya) $(\tau=2 \mu t$, with a mutation rate that has to be corrected over the total fragment length, i.e., $\times 3507 \mathrm{bp}$ ).

This age estimate for the seroresistant population is probably too high because of the importation by recombination of sequence diversity from the serosensitive population, which would artificially increase the genetic diversity of the expanding seroresistant group. Unfortunately, the pruned sequences cannot be evaluated by mismatch analyses, which cannot be applied because of the missing data that result from correcting for recombination.

An alternative approach to elucidating changes in the effective population size over time are so-called skyline plots, which are based on coalescent analyses of sequence data. Skyline plots that are parallel to the $X$-axis indicate a constant population size, and those that rise with time indicate population growth. The pattern of coalescent events for the genealogies of the concatenated housekeeping gene fragments provided further evidence for different evolutionary history of the serosensitive and seroresistant populations. For both populations, the genealogies were most consistent with a history of population growth rather than with constant effective population sizes (Table 3; Fig. 6). However, the processes shaping the demographic histories operated over different time scales. Assuming approximately clock-like evolution and the same mutation rate as E. coli, the age of the most recent common ancestor (MRCA) for the serosensitive population is $\sim 50 \mathrm{Myr}$ (raw data)-70 Myr (after purging for recombination). This is much older than
Table 2. Estimates of mutation rates $(\theta)$ and recombination rates $(\rho)$ per base

\begin{tabular}{|c|c|c|c|c|c|c|}
\hline & \multicolumn{2}{|c|}{$\theta$} & \multicolumn{2}{|c|}{$\rho$} & \multicolumn{2}{|c|}{$r / \mu$} \\
\hline & Sensitive & Resistant & Sensitive & Resistant & Sensitive & Resistant \\
\hline glybeta & 0.017 & 0.009 & 0.034 & $0.094^{\mathrm{a}}$ & 2.000 & 10.444 \\
\hline рра & 0.057 & 0.005 & $0.037^{\mathrm{a}}$ & $0.005^{\mathrm{a}}$ & 0.649 & 1.000 \\
\hline efp & 0.014 & 0.002 & $0.021^{\mathrm{a}}$ & $0.048^{a}$ & 1.500 & 24.000 \\
\hline trpE & 0.012 & NA & 0.030 & NA & 2.500 & NA \\
\hline fum $C$ & 0.024 & 0.004 & 0.009 & $0.022^{a}$ & 0.375 & 5.500 \\
\hline mutY & 0.028 & 0.013 & $0.072^{\mathrm{a}}$ & $0.016^{\mathrm{a}}$ & 2.571 & 1.231 \\
\hline$a b c Z$ & 0.024 & 0.014 & $0.029^{\mathrm{a}}$ & $0.006^{a}$ & 1.208 & 0.429 \\
\hline$a d k$ & 0.023 & 0.004 & 0.007 & $0.044^{\mathrm{a}}$ & 0.304 & 11.000 \\
\hline mean & 0.025 & 0.007 & 0.030 & 0.034 & 1.388 & 7.658 \\
\hline Concat. ${ }^{b}$ & 0.024 & 0.006 & 0.015 & 0.023 & 0.625 & 3.833 \\
\hline
\end{tabular}

Values for $\rho$ were obtained by dividing the per-locus recombination rate estimate from LDhat by the sequence length. (NA) Not available because of a lack of polymorphisms in order to implement the statistical test.

aSignificant estimates (at the $5 \%$ level).

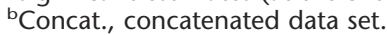




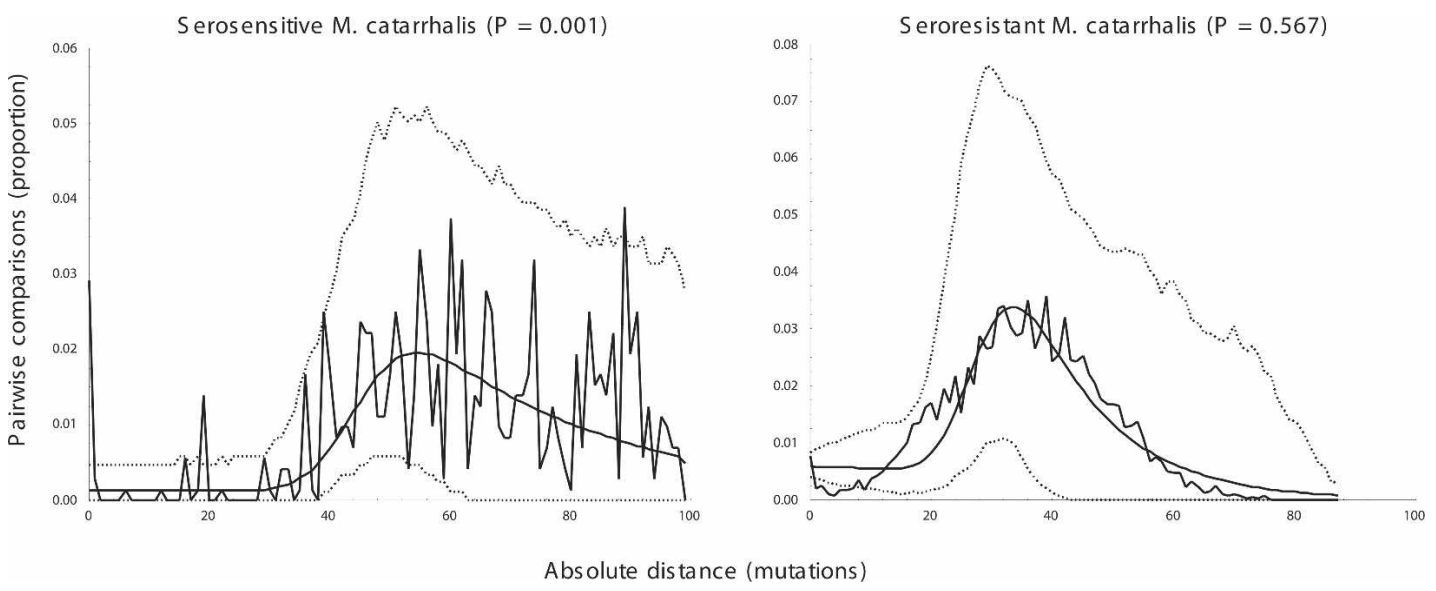

Figure 5. Mismatch frequency distributions of pairwise differences between concatenated sequences within serosensitive and seroresistant $M$. catarrhalis populations. The jagged plots correspond to the observed data. The smooth curves correspond to the predictions of a sudden expansion model whose $95 \%$ confidence intervals are indicated by dashed lines. P-values represent the probability that the raggedness of the simulated data set is equal to or greater than the observed data set.

cific IgG responses have been found in children and adults (Chen et al. 1999), reflecting ongoing host-bacteria interactions.

Our genealogical and coalescent-based analyses of multiple chromosomal loci have revealed that the two populations also differ greatly in their demographic histories. The serosensitive population displays much higher genetic diversity, a more moderate signal of expansion, and probably represents remnants of the ancestors of the modern M. catarrhalis that differentiated $\sim 50$ Mya. Note that $50 \mathrm{Myr}$ significantly predates the age of Homo sapiens, implying that the ancestral serosensitive population evolved in a different host, even though modern strains seem to be specific to humans. We do not know what hosts may have been infected by M. catarrhalis in the past, but it is likely that they were mammals because the closest relatives of $M$. catarrhalis (Moraxella bovis and Moraxella ovis) (Pettersson et al. 1998) infect cows and sheep. The present-day seroresistant population represents the consequences of a differential population expansion occurring over the last $4 \mathrm{Myr}$, during the Pliocene and Pleistocene and coincident with the rise of hominids. The size expansion of the seroresistant population possibly represents a second host shift, the acquisition of novel virulence genes, and/or strong selection for a particular clone. Our data show clearly that the emergence of $M$. catarrhalis is so old that it could not have evolved in the last 30-70 yr, the time period associated with the recent increased spread of seroresistant strains in hospitals. We are aware that the mutation rate used in this study is from a different bacterium (E. coli), but the age estimates for the seroresistant strains largely predate the age of antibiotics even with mutation rates that are one or two orders of magnitude greater.

The strong separations and long branch lengths between the two populations in phylogenetic trees (Fig. 3) indicate that they were isolated genetically for long time periods, possibly owing to geographical separation (allopatry) or to specialization for different hosts. More recently, the two lineages have come back into secondary genetic contact, probably through their global colonization of a common niche, the human respiratory tract. This secondary contact has resulted in gene flow between seroresistant and serosensitive strains, whereby diversity within housekeeping genes seems to have been imported into the seroresistant population from the serosensitive pool and uspA1 may have spread in the opposite direction by the selection and survival of particularly successful genotypes that escaped the host immune response.

\section{Homologous recombination and virulence}

It has long been recognized that recombination within bacterial species in nature is an important source of the diversity among modern isolates (Maynard Smith et al. 1993; Feil and Spratt 2001). The frequency of recombination is extremely variable, resulting in population structures that range from panmixia in Helicobacter pylori (Falush et al. 2001; Linz et al. 2007) and Neisseria meningitidis (Feil et al. 2001) to absolute clonality in Yesinia pestis (Achtman et al. 2004) and Mycobacterium tuberculosis (Baker et al. 2004). On the basis of preliminary data, Enright and McKenzie (1997) suggested that M. catarrhalis has a nonclonal structure due to extensive recombination. The analyses presented here show that homologous recombination is, indeed, frequent in the seroresistant population but rather rare in the serosensitive population. This striking contrast points to a link between sex, that is, homologous recombination, and virulence as also recently suggested in E. coli (Wirth et al. 2006). The association between the acquisition of novel genes by horizontal genetic exchange (HGT)

Table 3. Evaluation of alternative parametric demographic models for the genealogies of $M$. catarrhalis populations at eight concatenated housekeeping genes

\begin{tabular}{|c|c|c|c|c|}
\hline & \multicolumn{2}{|c|}{$\begin{array}{l}\text { Serosensitive } \\
\text { population }\end{array}$} & \multicolumn{2}{|c|}{$\begin{array}{l}\text { Seroresistant } \\
\text { population }\end{array}$} \\
\hline & Raw data & Purged & Raw data & Purged \\
\hline Highest likelihood model & plog & plog & pexpan & pexpan \\
\hline Constant model rejected & Yes** & Yes** & Yes** & No \\
\hline Expo model rejected & Yes* & Yes** & Yes** & No \\
\hline
\end{tabular}

The most likely model was compared with constant and exponential models. The absence of support for the different demographic models in the purged seroresistant population is likely due to its recent origin and lack of polymorphisms. For details, please refer to Pybus and Rambaut (2002). plog, piecewise logistic growth; pexpan, piecewise expansion growth; Expo, exponential.

${ }^{*} P<0.05$.

$* * P<0.01$.

\section{Genome Research}

www.genome.org 


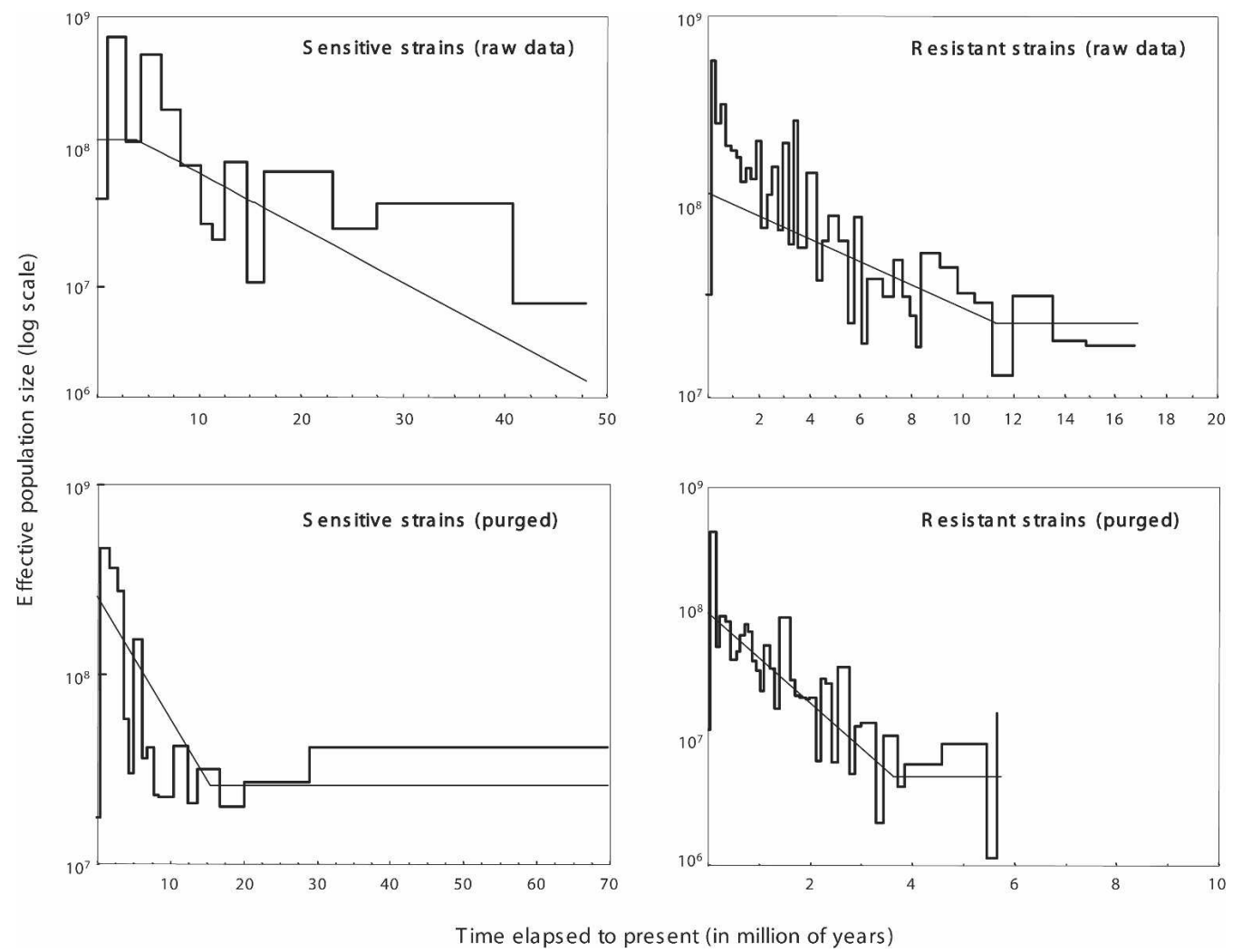

Figure 6. Estimated demographic histories of the serosensitive and seroresistant $M$. catarrhalis populations based on the concatenated sequences. The thicker lines are the generalized skyline plots. The approximate timescale assumes a mutation rate $(\mu)$ of $7.6 \times 10^{-10}$. The thinner, smooth curves represent the parametric ML demographic history (see Table 3).

and virulence is well established (Hacker and Kaper 2000). But are sex and virulence causally linked, or do these observations reflect indirect associations?

If the evolution of virulence were favored by a reduction of barriers to homologous recombination and HGT, then a single factor might be responsible for the link between sex and virulence. Reduced mismatch repair (MMR) increases both mutation and recombination rates, reducing genetic barriers between distantly related bacteria (Taddei et al. 1997b; Cooper and Lenski 2000; Denamur et al. 2000) and providing a foundation for rapid evolution (Cebula and LeClerc 1997; Sniegowski et al. 2000). Although not yet described in M. catarrhalis, mutator clones have been identified in epidemic serogroup A Neisseria meningitis (Jyssum 1968; Richardson et al. 2002), a species that is closely related to $M$. catarrhalis. If mutators were frequent in the seroresistant population, this would also affect our dating estimates, resulting in an overestimation of the age of this population. An involvement of MMR-deficient E. coli in the rapid emergence of antibiotic resistance and in the uptake of virulence genes within prokaryotes was first advocated in the mid-1990s (LeClerc et al. 1996). Mutators were recently shown to be frequent among Pseudomomas aeruginosa from the lungs of cystic fibrosis patients (Oliver et al. 2000) and among E. coli from chronic urinary tract infections (Labat et al. 2005). Thus, despite a lack of direct evidence, we speculate that elevated recombination in the seroresistant lineage reflects transient MMR-deficient mutators within this population. An evolutionary scenario for the two $M$. catarrhalis lineages reflecting this hypothesis is presented in Figure 7. According to the mutator scenario, it might have been ex- pected that the seroresistant grouping in Supplemental Figure S3 would display longer branches, which was not the case. This may simply reflect an overwhelming impact of recombination, which resulted in branch collapse.

Alternatively, the observed patterns could reflect a more prosaic mechanism whereby the evolution to a specialist involved a series of selective sweeps. In selective sweeps, there would be selection against MMR, and the link between sex and virulence would be indirect because of the evolutionary dynamics of the lineage rather than reflecting cause and effect.

\section{Toward a new paradigm}

The reduction of barriers to genetic exchange, perhaps by MMR deficiency, might also lead to more frequent import of virulence determinants. More virulent bacteria are more likely to cause invasive disease, with accompanying greater selection pressures due to the host's immune response, thus causing an "arms race" and increasing rates of evolution of both sex and virulence. This interpretation implies that increased recombination and mutation rates may be long-term features of pathogenic bacterial populations and that they affect the core genome and leave genome-wide signatures, even when they are transient properties of particular strains (Taddei et al. 1997a). Similar analyses could lend further support to the idea that sex and virulence are intimately related in microbes. Most pathogenic bacteria harbor a spectrum of virulence phenotypes, undergo at least limited levels of recombination, and are therefore preeminent candidates for such investigations. 

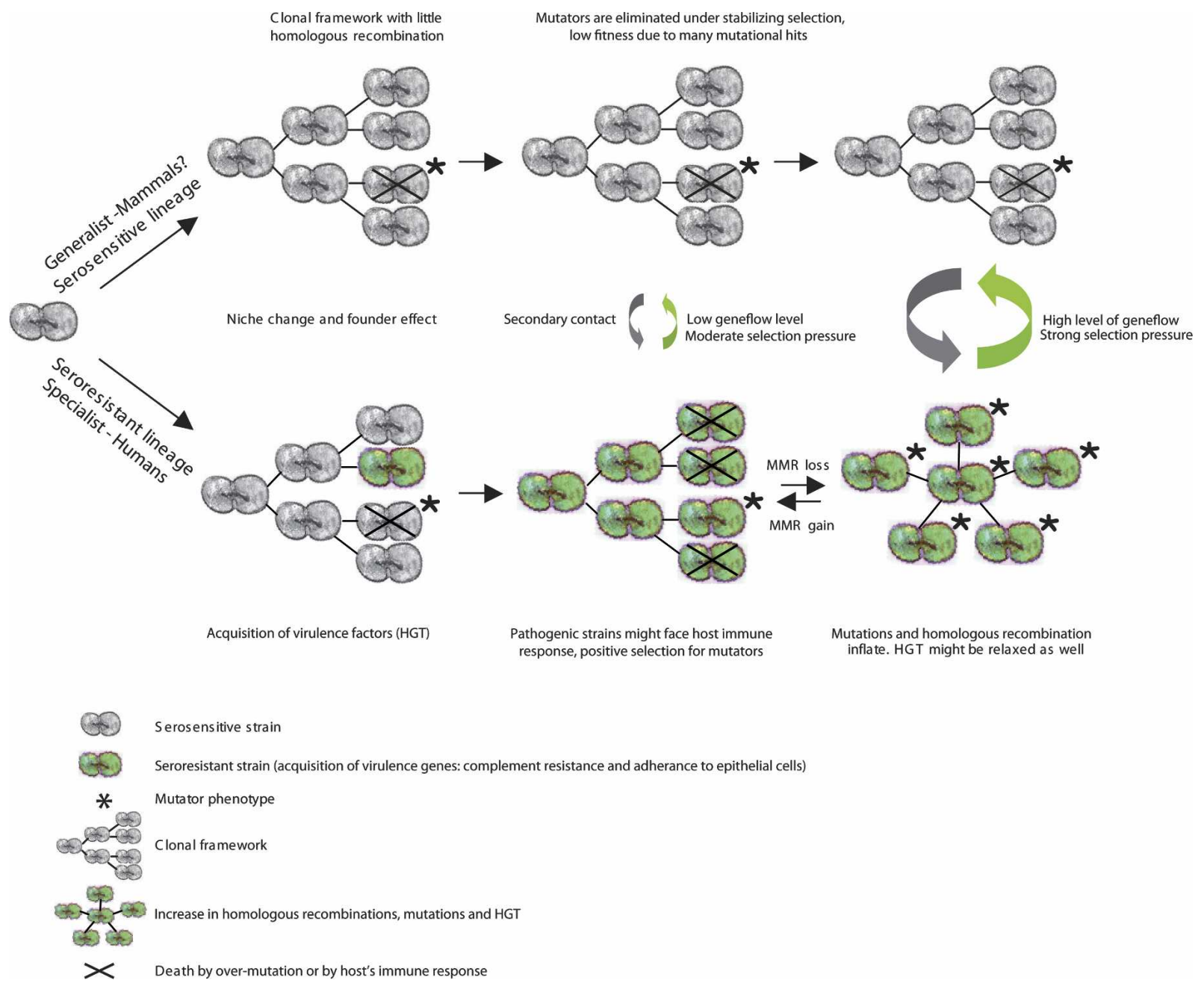

Figure 7. Model of evolutionary mechanisms that link sex and virulence. Virulence determinants are assumed to be introduced initially to a lineage by HGT. We deduce that (at least) two events were needed to convert an ancestral avirulent $M$. catarrhalis into virulent organisms. Within each population, mutators arise at low frequencies by random mutations. Mutator strains are transient within each population because they are eliminated because of lower fitness (crosses). But virulent strains face selection pressures for more rapid diversification in response to host immune defenses, resulting in higher frequencies of mutators in the seroresistant population. The frequency of transient mutators determines the population structure. At low frequencies, populations are largely asexual (clonal), whereas sex becomes more frequent with increasing mutator frequency. As a result, virulent bacteria are expected to possess patchy sexual structure within a generally asexual framework, while avirulent strains are largely clonal.

\section{Methods}

\section{Bacterial strains}

A total of 268 M. catarrhalis strains chosen at random from multiple healthy and diseased humans in diverse geographical areas were investigated in an attempt to study the genetic diversity of this bacterial species from an evolutionary perspective (Supplemental Table S1). A subsample of strains was tested for their sensitivity to complement-mediated lysis in a microtiter bactericidal assay that used 50\% pooled human serum (Verduin et al. 1995). Strains were subdivided into three categories: sensitive (S; $<3 \%$ survival of the bacteria after incubation for $1 \mathrm{~h}$ in $50 \%$ pooled human serum), resistant ( $\mathrm{R} ;>50 \%$ survival), and intermediate (I). Presence or absence of the uspA1 gene and assignment of 16S rRNA type are based on the data in Bootsma et al. (2000).

\section{DNA sequencing}

Eight gene fragments were amplified and sequenced from all isolates using the primers in Supplemental Table S2. PCR reactions were as follows: denaturation, $1 \mathrm{~min}$ at $94^{\circ} \mathrm{C}$; annealing, $1 \mathrm{~min}$ at $56^{\circ} \mathrm{C}$; extension, $1 \mathrm{~min}$ at $72^{\circ} \mathrm{C}$; 35 cycles. Independent amplicons were used to sequence both strands by an Applied Biosystems Prism 3700 automated sequencer with dRhodamine-labeled terminators (PE Applied Biosystems). The multilocus haplotypes are publicly available (http://web.mpiib-berlin.mpg.de/mlst).

\section{Phylogenetic analyses}

Sequences were aligned and trimmed to a uniform size by using SEQLAB and PILEUP (Wisconsin Package 9.1; GCG) and then concatenated. In order to gain an overview of the phylogenetic signal, we plotted pairwise transition and transversion distances against the total genetic distances using the DAMBE software package (Xia and Xie 2001), and we also tested our set of molecular sequences for substitution saturation (Xia et al. 2003). The best-fit model of DNA substitution and the parameter estimates used for tree reconstruction were chosen by performing hierarchical likelihood ratio tests implemented in PAUP* (Swofford 2003) and MODELTEST 1.05 (Posada and Crandall 1998). Phylo-

\section{Genome Research}

www.genome.org 
genetic trees were estimated for each data set with PAUP* incorporating the best-fit maximum-likelihood model of evolution. Additionally, MrBayes 2.01 (Huelsenbeck and Ronquist 2000) was used to sample phylogenies according to their posterior probabilities using Metropolis-coupled Markov Chain Monte-Carlo methods and to determine clade credibility values across a consensus phylogeny. Split decomposition analyses were performed with SplitsTree, version 4, by using LogDet distances, equal edge lengths, and 1000 bootstrap replicates.

\section{Tree purging}

In order to evaluate the impact of homologous recombination on bacteria phylogeny and demography, we used pruned sequences in which recombinant nucleotides were recoded as "N". The identification of recombinant nucleotides was based on the assignments to ancestral gene pools by the linkage model of STRUCTURE. Up to $30 \%$ of the polymorphic nucleotides were removed from the concatenated sequences depending on the strain.

\section{Demographic inferences}

To determine whether serosensitive or seroresistant populations underwent recent population expansions, we calculated mismatch distributions and compared these to predicted distributions from models of population expansion (Rogers 1995). For expanding populations, we converted the parameter tau $(\tau ;$ calculated from the mismatch distribution) to estimate time to the expansion $(t)$ using the equation $\tau=2 \mu t$, where $\mu$ is the neutral mutation rate for the locus. The confidence intervals of $\tau$ were calculated using a parametric bootstrap approach (Schneider and Excoffier 1999). Mismatch distributions and $\tau$ were calculated in ARLEQUIN 3.0 (Excoffier et al. 2005).

Mismatch analysis cannot account for missing data. We therefore also investigated the historical demography of each M. catarrhalis population by using generalized skyline plots (Strimmer and Pybus 2001), a graphical nonparametric estimate of effective population size, and evaluated alternative parametric demographic models by using GENIE (Version 3.0) (Pybus and Rambaut 2002). The input topology consisted of ML genealogies. The age of the evolutionary events was calculated based on a molecular clock rate of $7.6 \times 10^{-10} / \mathrm{yr}$ (Wirth et al. 2006). This clock rate is based on a divergence of $21.3 \%$ between the concatenated sequences of E. coli and Salmonella enterica, which diverged around 140 Mya (Ochman and Wilson 1987).

\section{Population genetic analyses}

Pairwise nucleotide diversity $(\pi)$ and the number of segregating sites (Watterson's $\theta$ ) were calculated with DnaSP, version 4.10 (Rozas et al. 2003). Three tests for selection were performed: Tajima's $D$, Fu's $F_{\mathrm{S}}$, and the $K_{\mathrm{a}} / K_{\mathrm{s}}$ ratio test.

We used the linkage model in STRUCTURE (Falush et al. 2003a) to identify groups with distinct allele frequencies (Falush et al. 2003b). This procedure assigns a probability of ancestry for each polymorphic nucleotide for a given number of groups, $K$, and also estimates $q$, the combined probability of ancestry from each of the $K$ groups for each individual isolate. We chose two groups for this report because repeated analyses (20,000 iterations, following a burn-in period of 10,000 iterations) with $K$ between 1 and 7 showed that the model probability increased dramatically between $K=1$ and $K=2$ and only slowly at higher values.

\section{Recombination and mutation}

The population recombination rate was estimated by a composite-likelihood method with LDhat (McVean et al. 2002). LDhat uses a parametric approach, based on the neutral coalescent, to estimate the scaled parameter $2 N_{\mathrm{e}} r$, where $N_{\mathrm{e}}$ is the effective population size and $r$ is the rate at which recombination events separate adjacent nucleotides. The gene conversion model $\mathrm{C}$ was used for the analysis of biallelic sites.

\section{Acknowledgments}

We thank Paul Bunje and Géraldine Bollmann for comments on the manuscript and Anthony Campagnari, Frits Mooi, Mario Vaneechoutte, Tone Toenjum, Mark Enright, Xavier Nassif, Sebastien Suerbaum, and Nicole Luke for providing M. catarrhalis isolates. This work was supported by SmithKline Beecham, a Deutsche Forschungsgemeinschaft Grant (WI 2710/1) to T.W., and the University of Konstanz.

\section{References}

Achtman, M., Morelli, G., Zhu, P., Wirth, T., Diehl, I., Kusecek, B., Vogler, A.J., Wagner, D.M., Allender, C.J., Easterday, W.R., et al. 2004. Microevolution and history of the plague bacillus, Yersinia pestis. Proc. Natl. Acad. Sci. 101: 17837-17842.

Aebi, C., Lafontaine, E.R., Cope, L.D., Latimer, J.L., Lumbley, S.L., McCracken Jr., G.H., and Hansen, E.J. 1998. Phenotypic effect of isogenic uspA1 and uspA2 mutations on Moraxella catarrhalis $035 \mathrm{E}$. Infect. Immun. 66: 3113-3119.

Baker, L., Brown, T., Maiden, M.C., and Drobniewski, F. 2004. Silent nucleotide polymorphisms and a phylogeny for Mycobacterium tuberculosis. Emerg. Infect. Dis. 10: 1568-1577.

Boel, E., Bootsma, H., de Kruif, J., Jansze, M., Klingman, K.L., van Dijk, H., and Logtenberg, T. 1998. Phage antibodies obtained by competitive selection on complement-resistant Moraxella (Branhamella) catarrhalis recognize the high-molecular-weight outer membrane protein. Infect. Immun. 66: 83-88.

Bootsma, H.J., van der Heide, H.G., van de Pas, S., Schouls, L.M., and Mooi, F.R. 2000. Analysis of Moraxella catarrhalis by DNA typing: Evidence for a distinct subpopulation associated with virulence traits. J. Infect. Dis. 181: 1376-1387.

Catlin, B.W. 1990. Branhamella catarrhalis: An organism gaining respect as a pathogen. Clin. Microbiol. Rev. 3: 293-320.

Cebula, T.A. and LeClerc, J.E. 1997. Hypermutability and homeologous recombination: Ingredients for rapid evolution. Bull. Inst. Pasteur 95: $97-106$.

Chen, D., Barniak, V., VanDerMeid, K.R., and McMichael, J.C. 1999 The levels and bactericidal capacity of antibodies directed against the UspA1 and UspA2 outer membrane proteins of Moraxella (Branhamella) catarrhalis in adults and children. Infect. Immun. 67: 1310-1316.

Cooper, V.S. and Lenski, R.E. 2000. The population genetics of ecological specialization in evolving Escherichia coli populations. Nature 407: 736-739.

Daoud, A., Abuekteish, F., and Masaadeh, H. 1996. Neonatal meningitis due to Moraxella catarrhalis and review of the literature. Ann. Trop. Paediatr. 16: 199-201.

Denamur, E., Lecointre, G., Darlu, P., Tenaillon, O., Acquaviva, C., Sayada, C., Sunjevaric, I., Rothstein, R., Elion, J., Taddei, F., et al. 2000. Evolutionary implications of the frequent horizontal transfer of mismatch repair genes. Cell 103: 711-721.

Enright, M.C. and McKenzie, H. 1997. Moraxella (Branhamella) catarrhalis clinical and molecular aspects of a rediscovered pathogen. J. Med. Microbiol. 46: 360-371.

Excoffier, L., Laval, G., and Schneider, S. 2005. Arlequin ver. 3.0: An integrated software package for population genetics data analysis. Evol. Bioinform. Online 1: 47-50.

Falush, D., Kraft, C., Taylor, N.S., Correa, P., Fox, J.G., Achtman, M., and Suerbaum, S. 2001. Recombination and mutation during long-term gastric colonization by Helicobacter pylori: Estimates of clock rates, recombination size, and minimal age. Proc. Natl. Acad. Sci. 98: 15056-15061.

Falush, D., Stephens, M., and Pritchard, J.K. 2003a. Inference of population structure using multilocus genotype data: Linked loci and correlated allele frequencies. Genetics 164: 1567-1587. 
Falush, D., Wirth, T., Linz, B., Pritchard, J.K., Stephens, M., Kidd, M. Blaser, M.J., Graham, D.Y., Vacher, S., Perez-Perez, G.I., et al. 2003b. Traces of human migrations in Helicobacter pylori populations. Science 299: 1582-1585.

Feil, E.J. and Spratt, B.G. 2001. Recombination and the population structures of bacterial pathogens. Annu. Rev. Microbiol. 55: 561-590.

Feil, E.J., Holmes, E.C., Bessen, D.E., Chan, M.S., Day, N.P., Enright, M.C., Goldstein, R., Hood, D.W., Kalia, A., Moore, C.E., et al. 2001 Recombination within natural populations of pathogenic bacteria: Short-term empirical estimates and long-term phylogenetic consequences. Proc. Natl. Acad. Sci. 98: 182-187.

Fu, Y.X. 1997. Statistical tests of neutrality of mutations against population growth, hitchhiking and background selection. Genetics 147: 915-925.

Hacker, J. and Kaper, J.B. 2000. Pathogenicity islands and the evolution of microbes. Annu. Rev. Microbiol. 54: 641-679.

Hager, H., Verghese, A., Alvarez, S., and Berk, S.L. 1987. Branhamella catarrhalis respiratory infections. Rev. Infect. Dis. 9: 1140-1149.

Harpending, H.C., Batzer, M.A., Gurven, M., Jorde, L.B., Rogers, A.R., and Sherry, S.T. 1998. Genetic traces of ancient demography. Proc. Natl. Acad. Sci. 95: 1961-1967.

Helminen, M.E., Maciver, I., Paris, M., Latimer, J.L., Lumbley, S.L., Cope, L.D., McCracken Jr., G.H., and Hansen, E.J. 1993. A mutation affecting expression of a major outer membrane protein of Moraxella catarrhalis alters serum resistance and survival in vivo. I. Infect. Dis. 168: 1194-1201.

Huelsenbeck, J.P. and Ronquist, F. 2000. MrBayes: Bayesian inferences of phylogeny. Bioinformatics 17: 754-755.

Johnson, M.A., Drew, W.L., and Roberts, M. 1981. Branhamella (Neisseria) catarrhalis a lower respiratory tract pathogen? J. Clin. Microbiol. 13: 1066-1069.

Jyssum, K. 1968. Mutator factor in Neisseria meningitidis associated with increased sensitivity to ultraviolet light and defective transformation. J. Bacteriol. 96: 165-172.

Labat, F., Pradillon, O., Garry, L., Peuchmaur, M., Fantin, B., and Denamur, E. 2005. Mutator phenotype confers advantage in Escherichia coli chronic urinary tract infection pathogenesis. FEMS Immunol. Med. Microbiol. 44: 317-321.

LeClerc, J.E., Li, B., Payne, W.L., and Cebula, T.A. 1996. High mutation frequencies among Escherichia coli and Salmonella pathogens. Science 274: $1208-1211$.

Linz, B., Balloux, F., Moodley, Y., Manica, A., Liu, H., Roumagnac, P., Falush, D., Stamer, C., Prugnolle, F., van der Merwe, S.W., et al. 2007. An African origin for the intimate association between humans and Helicobacter pylori. Nature 445: 915-918.

Maiden, M.C. 2006. Multilocus sequence typing of bacteria. Annu. Rev. Microbiol. 60: 561-588.

Maynard Smith, J., Smith, N.H., O'Rourke, M., and Spratt, B.G. 1993. How clonal are bacteria? Proc. Natl. Acad. Sci. 90: 4384-4388.

McVean, G., Awadalla, P., and Fearnhead, P. 2002. A coalescent-based method for detecting and estimating recombination from gene sequences. Genetics 160: 1231-1241.

Murphy, T.F. 1998. Lung infections. 2. Branhamella catarrhalis: Epidemiological and clinical aspects of a human respiratory tract pathogen. Thorax 53: 124-128.

Ochman, H. and Wilson, A.C. 1987. Evolution in bacteria: Evidence for a universal substitution rate in cellular genomes. J. Mol. Evol. 26: $74-86$.

Oliver, A., Canton, R., Campo, P., Baquero, F., and Blazquez, J. 2000 High frequency of hypermutable Pseudomonas aeruginosa in cystic fibrosis lung infection. Science 288: 1251-1254.

Patterson, T.F., Patterson, J.E., Masecar, B.L., Barden, G.E., Hierholzer Jr. W.J., and Zervos, M.J. 1988. A nosocomial outbreak of Branhamella catarrhalis confirmed by restriction endonuclease analysis. J. Infect. Dis. 157: 996-1001.

Pettersson, B., Kodjo, A., Ronaghi, M., Uhlen, M., and Tonjum, T. 1998. Phylogeny of the family Moraxellaceae by $16 \mathrm{~S}$ rDNA sequence analysis, with special emphasis on differentiation of Moraxella species. Int. J. Syst. Bacteriol. 48: 75-89.

Posada, D. and Crandall, K.A. 1998. MODELTEST: Testing the model of DNA substitution. Bioinformatics 14: 817-818.

Pritchard, J.K., Stephens, M., and Donnelly, P. 2000. Inference of population structure using multilocus genotype data. Genetics 155: 945-959.

Pybus, O.G. and Rambaut, A. 2002. GENIE: Estimating demographic history from molecular phylogenies. Bioinformatics 18: 1404-1405.

Richards, S.J., Greening, A.P., Enright, M.C., Morgan, M.G., and McKenzie, H. 1993. Outbreak of Moraxella catarrhalis in a respiratory unit. Thorax 48: 91-92.

Richardson, A.R., Yu, Z., Popovic, T., and Stojiljkovic, I. 2002. Mutator clones of Neisseria meningitidis in epidemic serogroup A disease. Proc. Natl. Acad. Sci. 99: 6103-6107.

Rogers, A.R. 1995. Genetic evidence for a Pleistocene population explosion. Evolution Int. J. Org. Evolution 49: 608-615.

Rozas, J., Sanchez-DelBarrio, J.C., Messeguer, X., and Rozas, R. 2003. DnaSP, DNA polymorphism analyses by the coalescent and other methods. Bioinformatics 19: 2496-2497.

Schneider, S. and Excoffier, L. 1999. Estimation of past demographic parameters from the distribution of pairwise differences when the mutation rates vary among sites: Application to human mitochondrial DNA. Genetics 152: 1079-1089.

Sniegowski, P.D., Gerrish, P.J., Johnson, T., and Shaver, A. 2000. The evolution of mutation rates: Separating causes from consequences. BioEssays 22: 1057-1066.

Strimmer, K. and Pybus, O.G. 2001. Exploring the demographic history of DNA sequences using the generalized skyline plot. Mol. Biol. Evol. 18: 2298-2305.

Stutzmann Meier, P., Heiniger, N., Troller, R., and Aebi, C. 2003 Salivary antibodies directed against outer membrane proteins of Moraxella catarrhalis in healthy adults. Infect. Immun. 71: 6793-6798.

Stutzmann Meier, P., Troller, R., Heiniger, N., Grivea, I.N., Syrogiannopoulos, G.A., and Aebi, C. 2005. Moraxella catarrhalis strains with reduced expression of the UspA outer membrane protein belong to a distinct subpopulation. Vaccine 23: 2000-2008.

Swofford, D.L. 2003. PAUP*-Phylogenetic analyses using parsinomy and other methods, version 4.0. Sinauer, Sunderland, MA.

Taddei, F., Matic, I., Godelle, B., and Radman, M. 1997a. To be a mutator, or how pathogenic and commensal bacteria can evolve rapidly. Trends Microbiol. 5: 427-428.

Taddei, F., Radman, M., Maynard-Smith, J., Toupance, B., Gouyon, P.H., and Godelle, B. 1997b. Role of mutator alleles in adaptive evolution. Nature 387: 700-702.

Tajima, F. 1989. Statistical method for testing the neutral mutation hypothesis by DNA polymorphism. Genetics 123: 585-595.

Verduin, C.M., Hol, C., Van Dijke, E., Faber, J.A., Jansze, M., Verhoef, J., and Van Dijk, H. 1995. Assessment of complement-mediated killing of Moraxella (Branhamella) catarrhalis isolates by a simple method. Clin. Diagn. Lab. Immunol. 2: 365-368.

Verduin, C.M., Hol, C., Fleer, A., van Dijk, H., and van Belkum, A. 2002 Moraxella catarrhalis: From emerging to established pathogen. Clin. Microbiol. Rev. 15: 125-144.

Wirth, T., Falush, D., Lan, R., Colles, F., Mensa, P., Wieler, L.H., Karch, H., Reeves, P.R., Maiden, M.C., Ochman, H., et al. 2006. Sex and virulence in Escherichia coli: An evolutionary perspective. Mol. Microbiol. 60: 1136-1151.

Xia, X. and Xie, Z. 2001. DAMBE: Software package for data analysis in molecular biology and evolution. J. Hered. 92: 371-373.

Xia, X., Xie, Z., Salemi, M., Chen, L., and Wang, Y. 2003. An index of substitution saturation and its application. Mol. Phylogenet. Evol. 26: $1-7$.

Zhang, L. and Li, W.-H. 2005. Human SNPs reveal no evidence of frequent positive selection. Mol. Biol. Evol. 22: 2504-2507.

Received November 14, 2006; accepted in revised form August 15, 2007.

\section{Genome Research}

www.genome.org 


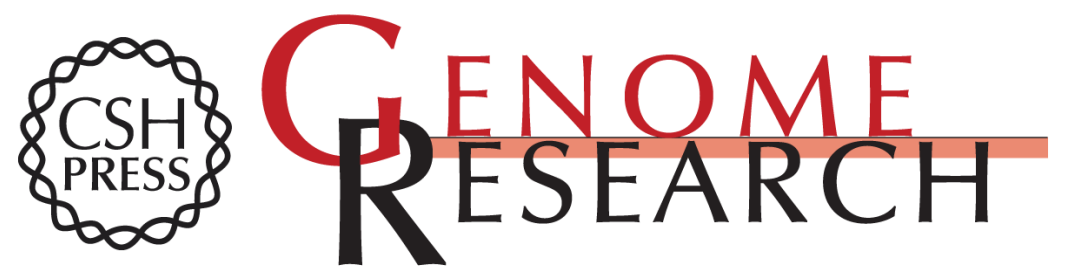

\section{The rise and spread of a new pathogen: Seroresistant Moraxella catarrhalis}

Thierry Wirth, Giovanna Morelli, Barica Kusecek, et al.

Genome Res. 2007 17: 1647-1656 originally published online September 25, 2007

Access the most recent version at doi:10.1101/gr.6122607

Supplemental Material

References

License

Email Alerting Service
http://genome.cshlp.org/content/suppl/2007/09/26/gr.6122607.DC1

This article cites 58 articles, 24 of which can be accessed free at: http://genome.cshlp.org/content/17/11/1647.full.html\#ref-list-1

Receive free email alerts when new articles cite this article - sign up in the box at the top right corner of the article or click here.

\section{Affordable, Accurate Sequencing.}

\section{gencove}

To subscribe to Genome Research go to: https://genome.cshlp.org/subscriptions 